\title{
Präferenzen von Menschen mit Pflegebedarf
}

\section{Eine explorative Analyse von Pflegedokumentation in verschiedenen Settings}

\author{
Mike Rommerskirch-Manietta ${ }^{1,2} \cdot$ Martina Roes $^{1,2} \cdot$ Tobias-Ingo Stacke $^{1,2} \cdot$ Christina Manietta $^{2}$. \\ Johannes-Michael Bergmann ${ }^{1,2} \cdot$ Daniel Purwins ${ }^{1,2}$
}

Eingegangen: 19. Mai 2020 / Angenommen: 9. Dezember 2020 / Online publiziert: 22. Januar 2021

(c) Der/die Autor(en) 2021

\section{Zusammenfassung}

Hintergrund Die Beachtung von Präferenzen des alltäglichen Lebens wird in der pflegerischen Versorgung mit einer Vielzahl von positiven Versorgungsergebnissen assoziiert. Derzeit ist unbekannt, ob in bundesdeutschen Pflegeeinrichtungen Präferenzen von Menschen mit Pflegebedarf systematisch erhoben werden, und inwiefern diese in der Pflegedokumentation auffindbar sind.

Ziel Das Ziel der Studie bestand darin zu untersuchen, welche und wie viele Präferenzen von Menschen mit Pflegebedarf in der Pflegedokumentation in verschiedenen pflegerischen Settings dokumentiert werden.

Methode Eine explorative Analyse von Pflegedokumentationen $(n=24)$ wurde in 4 Settings der pflegerischen Versorgung durchgeführt. Die erhobenen Daten wurden inhaltsanalytisch induktiv sowie quantitativ ausgewertet.

Ergebnis Es konnten 20 verschiedene Präferenzen identifiziert werden, die sich 5 Oberkategorien zuordnen ließen. Die Analyse zeigte in allen einbezogenen Settings, dass am häufigsten Präferenzen zu den Themen Pflege und Alltag in der Pflegedokumentation dokumentiert waren.

Schlussfolgerung Die Verschriftlichung von Präferenzen in der Pflegedokumentation ist in allen Settings unsystematisch, zugleich wird die Beachtung von Präferenzen des alltäglichen Lebens als relevant für die Pflege erachtet. Hier bietet die Verwendung eines Instruments zur systematischen Erfassung von Präferenzen die Möglichkeiten, einerseits die Sensibilisierung für dieses Thema zu stärken und andererseits die Zufriedenheit der Menschen mit Pflegebedarf zu steigern.

Schlüsselwörter Pflegeprozess · Psychosocial $\cdot$ Langzeitversorgung $\cdot$ Personenzentrierte Pflege $\cdot$ Dokumentenanalyse

\section{Preferences of people with care needs}

An exploratory analysis of nursing records in different nursing settings

\begin{abstract}
Background In nursing care, the consideration of preferences for everyday living is associated with a multitude of positive care outcomes. It is currently unknown whether the preferences of people with care needs are systematically assessed in German nursing facilities and to what extent these preferences can be found in the nursing records.

Aim The aim of the study was to investigate which and how many preferences of people with care needs are recorded in the nursing records in different nursing settings.

Method An explorative analysis of nursing records $(n=24)$ was conducted in four settings of nursing care. The collected data were inductively and quantitatively analyzed.
\end{abstract}

Mike Rommerskirch-Manietta

mike.rommerskirch@dzne.de

1 Deutsches Zentrum für Neurodegenerative Erkrankungen e. V. (DZNE), Standort Witten, Stockumer Str. 12, 58453 Witten, Deutschland
2 Fakultät für Gesundheit, Department für Pflegewissenschaft, Universität Witten/Herdecke, Witten, Deutschland 
Results A total of 20 different preferences were identified, which could be assigned to 5 superordinate categories. The analysis showed in all settings that preferences for the topics of care and everyday life were most frequently recorded in the nursing records.

Conclusion The recording of preferences in the nursing records is unsystematic in all settings, at the same time the consideration of preferences of everyday living is considered relevant for nursing. In this context, the use of an instrument for the systematic assessment of preferences offers the possibility to strengthen the sensitization to this topic and to increase the satisfaction of those with care needs.

Keywords Nursing process $\cdot$ Psychosocial $\cdot$ Person-centered care $\cdot$ Long-term care $\cdot$ Documentation analysis

\section{Hintergrund}

Alle Maßnahmen in Gesundheitseinrichtungen sind auf das Individuum und seine bzw. ihre pflegerischen Bedarfe und Präferenzen hin auszurichten (Eberhardt 2018). Eine Voraussetzung dafür ist, dass professionell Pflegende über Kenntnisse verfügen, die sich nicht nur auf pflegerische Bedarfe beziehen, sondern auch auf die für das Individuum relevanten Wünsche und Erwartungen erstrecken und professionell Pflegende diese ebenso bei der Versorgung berücksichtigen (Kesselring 2013). Die Individualität der Menschen mit Pflegebedarf kommt unter anderen in den Wünschen und Vorlieben bezogen auf die Bereiche des alltäglichen Lebens zum Ausdruck (Brummel-Smith et al. 2016). Die Umsetzung einer die Präferenzen der Menschen mit Pflegebedarf berücksichtigenden Versorgung ist ein wesentliches Qualitätsmerkmal für eine professionelle pflegerische Versorgung (Koren 2010; Gordon und Watts 2011).

Als Präferenzen des alltäglichen Lebens werden Wünsche, bezogen auf Bedürfnisse, inklusive der Art und Weise ihrer Erfüllung, verstanden (Abbott et al. 2018). Die Berücksichtigung von Präferenzen des alltäglichen Lebens in der pflegerischen Versorgung wird mit einer Vielzahl von positiven Versorgungsergebnissen assoziiert. So wird u.a. davon berichtet, dass sich das Wissen über sowie die Berücksichtigung von Präferenzen in der pflegerischen Versorgung oder bei Aktivitätsangeboten positiv auf den Ernährungszustand, den Kontinenzstatus sowie das Wohlbefinden von Menschen mit Pflegebedarf auswirken kann (Van Haitsma et al. 2015; Simmons und Schnelle 2004; Thompson und Smith 1998). In den Vereinigten Staaten von Amerika stehen verschiedene Instrumente zur strukturierten Erfassung von Präferenzen des alltäglichen Lebens von Menschen mit Pflegebedarf zur Verfügung (Van Haitsma et al. 2013; Housen et al. 2009; Cohen-Mansfield und Jensen 2007). Exemplarisch sei an dieser Stelle auf das Instrument Preferences for Everyday Living Inventory (PELI) (Van Haitsma et al. 2013) verwiesen. Zudem sind seit dem Jahr 2010 in einigen Bundesstaaten der USA staatlich zertifizierte Einrichtungen der stationären Langzeitversorgung verpflichtet, definierte Präferenzen des alltäglichen Lebens zu erfassen (Saliba und
Buchanan 2008; Saliba et al. 2012). In Deutschland existieren bisher keine für den deutschen Kontext entwickelten oder übersetzten Instrumente zur systematischen Erfassung von Präferenzen (Stacke et al. 2020a, b). Insofern ist unbekannt, inwieweit in deutschen Pflegeeinrichtungen dennoch Präferenzen des alltäglichen Lebens von Menschen mit Pflegebedarf in den verschiedenen Settings der pflegerischen Versorgung von professionell Pflegenden erhoben bzw. in der Pflegedokumentation verschriftlicht werden.

\section{Ziel und Fragestellungen}

Ziel der Studie war es, einen ersten explorativen Einblick in die Erfassung und Dokumentation von Präferenzen des alltäglichen Lebens von Menschen mit Pflegebedarf in 4 Settings der pflegerischen Versorgung zu erhalten. Folgende 2 Fragestellungen wurden formuliert: (1) Welche Präferenzen des alltäglichen Lebens von Menschen mit Pflegebedarf werden in der Pflegedokumentation verschriftlicht, und wie oft? (2) Existiert ein Unterschied in der Anzahl der dokumentierten Präferenzen des alltäglichen Lebens zwischen den unterschiedlichen Settings der pflegerischen Versorgung?

\section{Methode}

Für die durchgeführte Studie wurde ein Querschnittdesign mit einem explorativen Ansatz gewählt, um einen ersten Einblick in die Dokumentationspraktiken, bezogen auf die Präferenzen, zu erhalten. Bei den erhobenen Daten handelte es sich um Routinedaten, die den Sekundärdaten zuzuordnen sind (AGENS 2014). Diese konnten mithilfe eines selbst erstellten strukturierten Erfassungsbogen erhoben und für die Dokumentationsanalyse verwendet werden.

\section{Stichprobe und Rekrutierung}

Drei Einrichtungen der stationären Langzeitversorgung, 6 ambulante Pflegedienste, 2 Tagespflegeeinrichtungen und eine Wohngruppe für Menschen mit Pflegebedarf konnten 


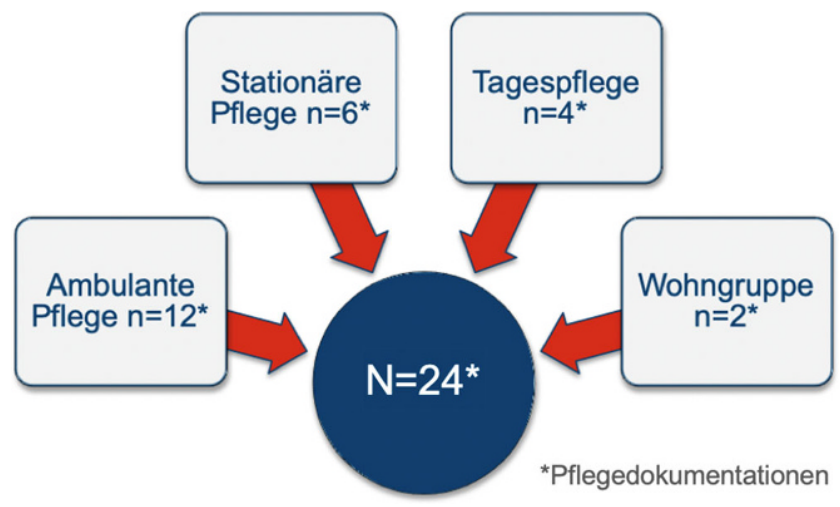

Abb. 1 Zusammensetzung der Stichprobe der zu analysierenden Pflegedokumentationen aus den jeweiligen Settings

mithilfe kooperierender Praxispartner*innen des Deutschen Zentrums für Neurodegenerative Erkrankungen (DZNE) für die Teilnahme an der Studie gewonnen werden $(n=12)$. Für die Ziehung der Stichprobe wurden die teilnehmenden Einrichtungen bzw. Dienste gebeten, jeder Pflegedokumentation von Menschen mit Pflegbedarf, die mindestens eine Leistung des Sozialgesetzbuches XI in Anspruch nehmen, einen Kode zuzuordnen. So wurde ausgeschlossen, dass sich die Pflegedokumentation ausschließlich auf die medizinische Behandlungspflege (SGB-V-Leistungen) bezog. Die von den Einrichtungen bzw. Diensten erstellten pseudonymisierten Kodelisten wurden elektronisch an das DZNE versandt. Anschließend wurde aus diesen Listen pro Einrichtung computergestützt eine zufällige Auswahl von je 2 Dokumentationen gezogen. Die Stichprobe bestand somit aus insgesamt 24 Pflegedokumentationen (Abb. 1).

\section{Erhebungsinstrument und Datenerhebungsvorgang}

Für die Datenerhebung wurde im Vorfeld ein strukturierter Erfassungsbogen zur Erhebung von Präferenzen im ,portable document format" (PDF) erstellt. Für die Entwicklung des Erfassungsbogens wurde bei den teilnehmenden Einrichtungen im Vorfeld erfragt, welches Dokumentationssystem bei ihnen verwendet wird. Auf Grundlage der Antworten wurden jeweils ein Erfassungsbogen für das, im Auftrag des Bundesministerium für Gesundheit entwickelte, Strukturmodell und die strukturierte Informationssammlung (SIS) (Beikirch et al. 2014) sowie ein Erhebungsbogen, strukturiert nach den Aktivitäten des täglichen Lebens (Krohwinkel 2013), erstellt. Diese 2 Versionen des strukturierten Erfassungsbogens sind für die Studie entwickelt worden und waren an den Schritten des Pflegeprozesses orientiert, die sich in verschiedenen Dokumentationsformularen wiederfinden (Informationssammlung, Maßnahmen-/bzw. Pflegeplanung, Berichteblatt und Evaluation sowie - falls vorliegend - Beratungsblatt in der ambulanten Pflege). Dazu wurden die Interviewpartner*innen gebeten, die für die Beantwortung der Fragen benötigten Textpassagen ohne Nennung von personenbezogenen Daten aus der Pflegedokumentation vorzulesen. Ziel war es, möglichst alle dokumentierten Präferenzen unabhängig vom jeweiligen Dokumentationsabschnitt innerhalb der Dokumentation zu erfassen. Im Vorfeld der Erhebungen erfolgte ein Pretest des Erfassungsbogens an jeweils einer Dokumentation mit dem Dokumentationssystem der SIS sowie einer Dokumentation, strukturiert nach den Aktivitäten des täglichen Lebens. Als Ergebnis wurden Anpassungen an der Fragenabfolge durchgeführt. Für die Datenerhebungen wurde im Sinne einer offenen Befragung, und orientiert am strukturierten Erfassungsbogen, ein bzw. eine professionell Pflegender/ Pflegende vor Ort zu den dokumentierten Präferenzen der stichprobenartig ausgewählten Menschen mit Pflegebedarf interviewt. Das heißt, die Forschenden erfragten Wünsche, Vorlieben, Erwartungen sowie Abneigungen der Menschen mit Pflegebedarf, die in den jeweils vorliegenden Pflegedokumentationen notiert waren. Die Antworten wurden von den Forschenden computergestützt mithilfe eines Tablets notiert.

\section{Ethik und Datenschutz}

Bei der durchgeführten Studie handelt es sich um die Erfassung und Auswertung von Sekundärdaten. Gemäß der Leitlinie Gute Praxis Sekundärdatenanalyse (AGENS 2014) war die Konsultation einer Ethikkommission für das Forschungsvorhaben nicht erforderlich. Zur Wahrung des Datenschutzes wurden den Forschenden elektronisch pseudonymisierte Listen mit den einzelnen Kodes der Pflegedokumentationen von den teilnehmenden Einrichtungen bzw. Diensten zur Verfügung gestellt. Zugriff auf die elektronischen pseudonymisierten Kodelisten hatten nur die Projektmitarbeitenden des DZNE. Die Kodelisten wurden auf einem geschützten Serverbereich des DZNE gespeichert und nach Projektende vom Server gelöscht. Durch das gewählte Vorgehen bei der Datenerhebung (s. unten) wurde sichergestellt, dass die Forschenden ausschließlich die für die Beantwortung der Fragestellungen notwendigen Informationen erhielten. Von den professionellen Pflegenden wurde eine informierte Einwilligung für die Studie eingeholt. Darüber hinaus wurde darauf hingewiesen, dass nicht die Qualität der pflegerischen Versorgung im Fokus der Erhebung steht und folglich keine Nachteile durch die Teilnahme an der Studie zu befürchten sind. Dem Datenschutz folgend wurden weder die Namen der für die Dokumentation verantwortlichen Pflegefachpersonen noch die der interviewten Pflegenden erhoben. Ebenfalls wurde auf eine Audioaufzeichnung der Interviews verzichtet. 


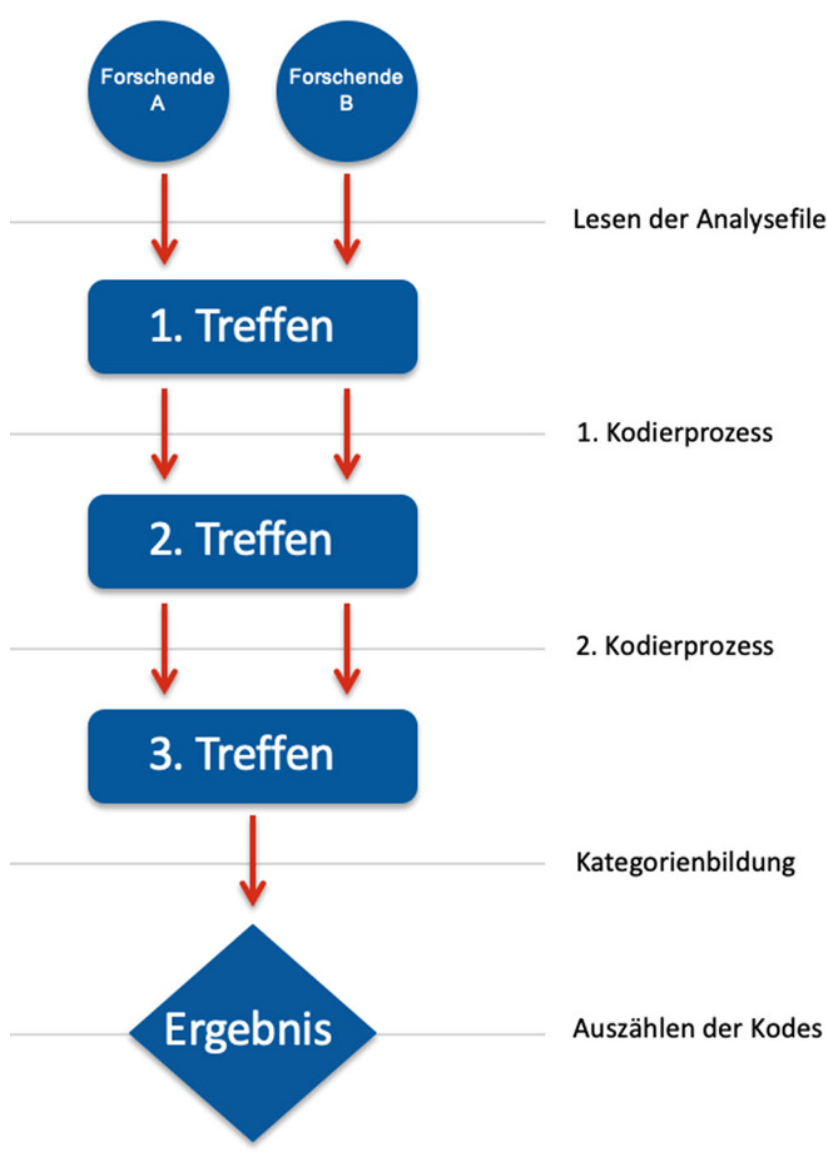

Abb. 2 Schritte des durchgeführten induktiven Kodierverfahrens

\section{Datenauswertung}

Für die Datenauswertung wurden qualitative sowie quantitative Methoden verwendet. In Anlehnung an Morgan (1993) wurde eine induktive Inhaltsanalyse durchgeführt (Abb. 2).

In einem ersten Schritt wurden die verschiedenen erstellten Analysedokumente von 2 Forschenden in ihrer Gesamtheit jeweils Satz für Satz gelesen. Dies erfolgte, um einen ersten Einblick und Überblick getrennt voneinander zu erhalten sowie eine Vertrautheit mit dem Datenmaterial zu erreichen. Daran anschließend tauschten sich die Forschenden in einem ersten Treffen über spontane Überlegungen, Auffälligkeiten sowie Ideen aus, die beim ersten Lesen des Datenmaterials aufkamen. In einem weiteren Schritt wurde jedes Analysedokument von den 2 Forschenden zunächst getrennt voneinander induktiv kodiert. Im Anschluss daran wurden die Analyseergebnisse in einem weiteren Treffen diskutiert, und es wurde eine zweite Phase des Kodierens durchgeführt. Nachdem diese zweite Phase des Kodierens abgeschlossen war und alle Analysedokumente rekodiert waren, konnte nur eine geringe Abweichung, bezogen auf die inhaltliche Übereinstimmung, der getrennt voneinander durchgeführten Kodierungen festgestellt werden. Folglich konnte ein Konsens mithilfe einer Diskussion innerhalb eines dritten Treffens zwischen den 2 Forschenden erreicht werden. Für den Fall, dass kein Konsens zwischen beiden Forschenden hätte gefunden werden können, wäre das gesamte Projektteam in die Konsensfindung miteingebunden worden. Anschließend wurden von den 2 Forschenden anhand des Datenmaterials induktiv Oberkategorien gebildet und die verschiedenen Kodierungen diesen aufgrund ihrer Inhalte zugeordnet.

Im Anschluss daran wurden für jedes Pflegesetting und jede Oberkategorie die Kodierungen quantifiziert. Für einen quantitativen Vergleich der dokumentierten Präferenzen in den verschiedenen pflegerischen Settings wurden der Median, der Interquartilsabstand, die Spannweite sowie das Minimum und das Maximum mithilfe von SPSS Vers. 21 berechnet.

\section{Ergebnisse}

Nachfolgend werden die Ergebnisse der qualitativen sowie quantitativen Analyse getrennt voneinander vorgestellt.

\section{Ergebnisse der qualitativen Analyse}

Insgesamt wurden durch die induktive Inhaltsanalyse 20 Präferenzen identifiziert, die sich 5 Kategorien zuordnen ließen (Abb. 3). Diese werden im Folgenden näher erläutert.

\section{Kategorie Pflege und Alltag}

Dieser Kategorie konnten 7 Themen zugeordnet werden, die von den Interviewpartnern/Interviewpartnerinnen als Präferenzen in den ausgewählten Dokumentationen identifiziert wurden. Es handelt sich um die Themen: Ernährung, Bewegung, Schlafen, Körperpflege, Bekleidung sowie Haushaltsführung und Tagesstruktur.

Aussagen zu Präferenzen, die dem Thema Ernährung zugeordnet werden konnten, bezogen sich zum einen auf die Ausgestaltung der Mahlzeiten (u. a. Zeitraum, Bedeutung einzelner Mahlzeiten, Einbezug, Art der Zubereitung) sowie auf die Umgebungsgestaltung. Dies zeigte sich in der Dokumentation beispielsweise wie folgt: „Frühstück unter den Mahlzeiten besonders wichtig, sie sagt immer: Morgens wie ein Kaiser, mittags wie ein Edelmann und abends wie ein Bettler" (B103) oder auch „Kaffee muss immer sehr heiß sein, Kaffeekanne muss vor Nutzung immer heiß ausgespült werden und parallel zum Brötchen serviert werden " (G128). Zum anderen wurden Präferenzen identifiziert, die sich auf die Abneigungen und Vorlieben von Nahrungsmitteln und Getränken bezogen. In diesem Zusammenhang wurde beispielsweise „mag Zwiebeln, aber keinen Pfeffer" 
Abb. 3 Identifizierte Präferenzen und deren Zuordnung zu den Oberkategorien

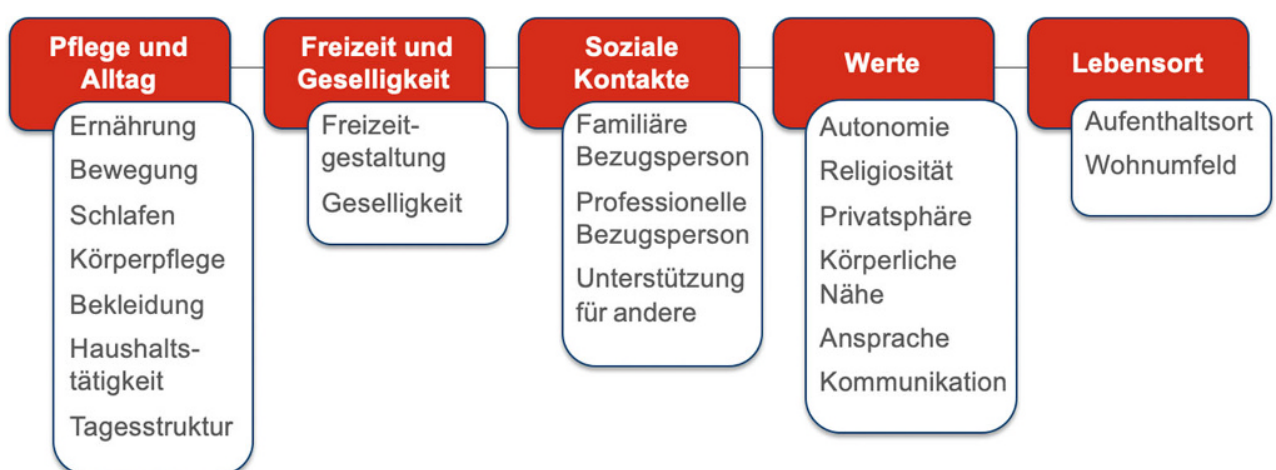

(B105) oder auch , liebt Pflaumenkuchen“ (S101) beschrieben.

Aussagen zu präferierten sportlichen Aktivitäten und Bewegungsarten wurden dem Thema Bewegung zugeordnet. So wurden die Textpassagen ,macht gerne Übungen bei der Morgengymnastik“ (D309), ,geht tanzen“ (K325) sowie ,geht gerne spazieren“ (D105), und ,fährt gerne Fahrrad" (T145) identifiziert. Zudem wurden Dokumentationseinträge zu dem präferierten Bewegungsumfang, wie beispielsweise ,bewegt sich insgesamt viel“" (K533), in der Dokumentation identifiziert.

Aussagen zu Präferenzen wie ,,liegt gerne auf der linken Seite“ (S159) oder auch „Zimmer muss komplett dunkel sein zum Schlafen “ (K132) wurden dem Thema Schlafen zugeordnet und umfassten auch Aussagen zur Positionierung im Bett, zum Schlafort sowie zur Gestaltung des Bettes oder Raumes.

Aussagen zu Präferenzen mit Bezug zur persönlichen Hygiene und deren Ausgestaltung, dem Zeitpunkt, den Hygieneartikeln, Pflegeprodukten und Hilfsmittel sowie den Zuständigkeiten wurden im Themenkomplex Körperpflege zusammengefasst. So wurden z.B. Aussagen wie „duscht gerne etwas wärmer“ (T124) und ,nicht inkontinent ... trägt auf Wunsch Slipeinlagen, die sie sich selbst organisiert" (T145) in der Pflegedokumentation als Präferenz identifiziert.

Aussagen zu Präferenzen, die dem Thema Bekleidung zugeordnet wurden, beziehen sich auf Aspekte wie die Art des Kleidungsstücks, Material der Kleidung, Passform sowie Auswahl und Wechsel der Kleidung. Die Interviewpartner*innen identifizierten schriftliche Aussagen wie „Kleidung soll nicht gewechselt werden“ (D203), „liebt Hosen“ (T145) sowie „Rosa Bademantel - verbringt den Tag darin" (K132) als Präferenzen.

Ergänzend wurden Aussagen zu Präferenzen identifiziert, wie beispielsweise ,hilft gerne in der Haushaltsführung “(B105), ,beteiligt sich nicht gerne an hauswirtschaftlichen Tagesangeboten“ (D312) sowie „mag nicht gerne kochen" (B103). Diese wurden dem Thema Haushaltsführung zugeordnet. Auffällig war hier, dass die
Nennung dieser Präferenzen in der Regel im Kontext der Biografie der Menschen mit Pflegebedarf oder mit Bezug auf das hauswirtschaftliche Beschäftigungsangebot der verschiedenen Einrichtungen erfolgte.

Auch wurden schriftliche Aussagen als Präferenz identifiziert, wie beispielsweise ,steht gerne besonders früh auf“ (T145). Diese bezogen sich insgesamt auf gewünschte Aufsteh- und Ruhezeiten und wurden dem Thema Tagesstruktur zugeordnet.

\section{Kategorie Freizeit und Geselligkeit}

Es wurden Präferenzen genannt, die 2 Themen zugeordnet werden konnten.

Dokumentierte Präferenzen, die sich auf Aktivitäten beziehen, die von der Einrichtung angeboten werden, oder auch Aktivitäten, die der Mensch mit Pflegebedarf noch selbstständig durchführt, wurden zu dem Thema Freizeitgestaltung zusammengefasst. So wurde z. B. im Bereich der Freizeitgestaltung von „schaut TV, liest Krimis, telefoniert mit der Tochter, schaut Tierdokus“ (K132) und „werken und Gesellschaftsspiele nicht“ (K208) berichtet.

Beschreibungen von Präferenzen, die sich auf das Gemeinschaftsleben der Menschen mit Pflegebedarf in den verschiedenen teilnehmenden Einrichtungen sowie in deren eigenen Zuhause auszeichneten, wurden dem Thema Geselligkeit zugeordnet. Zusätzlich wurden Textstellen zum Familienleben und zu dessen Ausgestaltung identifiziert. Unter anderem wurden Aussagen wie ,lehnt Kontakt zu Mitbewohner ab“ (K132), „mag nicht, wenn Bewohner ihr zu nahekommen (dann schnell gereizt)“ (D242) oder auch „isst gerne mit der Tochter ein Eis zusammen“ (K533) dokumentiert.

\section{Kategorie soziale Kontakte}

Die von den Interviewpartnern/Interviewpartnerinnen identifizierten Aussagen lassen sich 3 Themen zuordnen.

Präferenzen, die im Zusammenhang mit der Bedeutung der Familie sowie dem Kontakt und der Beziehung 
zu einzelnen Familienmitgliedern standen, wurden dem Thema familiäre Bezugspersonen zugeordnet. So wurde beispielsweise „Zusammenhalt in der Familie ... stolz auf Sohn“ (B103) oder „Guter Kontakt zur Tochter ... genießt Besuche" (B105) in der Pflegedokumentation beschrieben. Zudem wurden Aktivitäten mit der Familie, die Unterstützung durch Familienmitglieder sowie die Ansprechperson für die Pflegenden aufgeführt.

Dokumentierte Aussagen wie ,fixiert auf einzelne Pflegende (insbesondere die Wohnbereichsleitung, Betreuungsassistenten)" (D203) wurden dem Thema professionelle Bezugspersonen zugeordnet.

Dokumentierte Präferenzen, wie , kümmert und hilft Mitbewohnern, fühlt sich verantwortlich und zugleich überfordert“ (B103) sowie „ist gerne freiwillig für andere tätig; in der Tagespflege übernimmt er z. B. die Position des Heimbeirats und gibt anderen Bewohnern/Gästen Informationen " (G115), wurden dem Thema Unterstützung für andere zugeordnet.

\section{Kategorie Werte}

Diese Oberkategorie umfasst 6 Themen: Autonomie, Religiosität, Privatsphäre, körperliche Nähe, Ansprache und Kommunikation.

Dokumentierte Aussagen, die sich einerseits auf eine selbstbestimmte Lebensführung sowie die Lebensorte beziehen und andererseits auf die Selbstbestimmung im Bezug zur professionellen Pflege, wurden zu dem Thema Autonomie zusammengefasst. Identifiziert wurden beispielsweise dokumentierte Aussagen wie ",nur weibliches Pflegepersonal gewünscht für Intimpflege“ (T124) und „Duschtage auf ihren Wunsch hin festgelegt (Mo, Di, Do, Fr)“ (T145).

Außerdem wurden dokumentierte Präferenzen identifiziert, die sich auf die Glaubensrichtung und Glaubenspraktiken, wie z.B. „wünscht keine Seelsorge für sich“ (T145) oder auch „,katholisch ... Tischgebet ist nicht wichtig ... zur Weihnachtszeit gerne in der Kirche/mag gerne größere (religiöse) Feste" (G115) bezogen. Diese wurden dem Thema Religiosität zugeordnet.

Das Thema Privatsphäre beinhaltet dokumentierte Präferenzen wie „... Tischmanieren“ (B105), „Haus/Eigentum ...“ (B103) oder auch „Freundlichkeit“ (B103).

Aussagen zu dokumentierten Präferenzen hinsichtlich der Art und Weise von Distanz und Nähe, der Körperstellen, die für Intimität stehen, sowie den Personen, mit denen der Mensch mit Pflegebedarf Nähe erleben möchte, wurden dem Thema körperliche Nähe zugeordnet. Demgemäß wurde unter anderen dokumentiert „mag von ihrem Lebensgefährten nicht auf das Ohr geküsst werden (Tinnitus)“ (D242) oder auch „Massage und insbesondere Handmassage “ (K326).
Dem Thema Ansprache wurden Präferenzen zugeordnet, wie beispielsweise „möchte mit Vornamen angesprochen werden " (B105).

Die in der Dokumentation identifizierten Präferenzen, die zum Thema Kommunikation zusammengefasst wurden, beziehen sich auf Aussagen wie ,spricht gerne rheinisches Plattdeutsch mit anderen " (G128) sowie auf Hinweise auf Gesprächspersonen oder Themen für die Kommunikation mit anderen.

\section{Kategorie Lebensort}

In dieser Kategorie befinden sich Präferenzen, die sich auf die 2 Themen Aufenthaltsort und Wohnumfeld beziehen.

Aussagen zu dokumentierten Präferenzen, die dem Thema Aufenthaltsort zugeordnet wurden, beziehen sich einerseits auf im Haus oder in der Wohnung sein. Beispielsweise wurde dokumentiert „sitzt gerne neben Bücherregal im Sessel“ (K208) oder „Lieblingsplatz beim Essen neben dem Fenster" (T124). Andererseits auf das Nach-draußenGehen; hier wurden beispielsweise die Einträge ,gerne an der frischen Luft" (T145) sowie ,gerne draußen oder mag Aktivitäten draußen" (D309) identifiziert.

Es wurden Aussagen ausgemacht, wie z. B. , legt sich ein Glas und Besteck und Teller auf den Nachtisch, falls sie etwas essen möchte in der Nacht" (K132) sowie ,Wohnraumgestaltung jahreszeitlich“ (K325) oder auch „Nachtschrank soll nicht verschoben werden“ (D203), die zu dem Thema Wohnumfeld zusammengefasst wurden.

\section{Ergebnisse der quantitativen Analyse}

Insgesamt konnten 149 dokumentierte Aussagen bezüglich der Kategorie Pflege und Alttag, 95 Aussagen der Kategorie Freizeit und Geselligkeit, 31 Aussagen bezogen auf soziale Kontakte, 72 Einträge zu Werten sowie 30 Aussagen bezogen auf den Lebensort gezählt werden. Die Verteilung der dokumentierten Aussagen, die pro Kategorie und Setting als Präferenzen von den Interviewpartnern/ Interviewpartnerinnen identifiziert wurden, ist Tab. 1 zu entnehmen.

Aussagen, die den Kategorien Pflege und Alltag sowie Freizeit und Geselligkeit zugeordnet werden konnten, stellten die am häufigsten dokumentierten Präferenzen $(n=149$ bzw. $n=95)$ dar. In Kontrastierung dieser beiden Kategorien, unter Berücksichtigung der verschiedenen Settings, ist die höchste Anzahl der Präferenzen in der Kategorie Pflege und Alltag in der ambulanten Pflege $(n=88)$ vorzufinden. Im Vergleich zu anderen Settings wurden in der ambulanten und stationären Pflege Präferenzen zu Freizeit und Geselligkeit nahezu gleich oft genannt $(n=34$ bzw. $n=35$ ). 
Tab. 1 Gesamtübersicht der quantitativen Verteilung der dokumentierten Inhalte pro Kategorie und Setting

\begin{tabular}{|c|c|c|c|c|c|}
\hline \multirow[t]{2}{*}{ Kategorie } & \multicolumn{4}{|c|}{ Setting der pflegerischen Versorgung } & \multirow[b]{2}{*}{ Gesamt } \\
\hline & Ambulant & Stationär & Tagespflege & Wohngruppe & \\
\hline Pflege und Alltag (n) & 88 & 22 & 17 & 22 & 149 \\
\hline Median & 6,50 & 3,00 & 4,00 & 11,00 & \\
\hline IQR & $5,00-10,00$ & $0,75-7,25$ & $2,00-6,75$ & a & \\
\hline Spannweite & 17 & 8 & 5 & 6 & \\
\hline Min.-Max. & $0-17$ & $0-8$ & $2-7$ & $8-14$ & \\
\hline Freizeit und Geselligkeit (n) & 34 & 35 & 16 & 10 & 95 \\
\hline Median & 2,50 & 5,50 & 4,00 & 5,00 & \\
\hline IQR & $1,25-4,50$ & $3,25-9,25$ & $3,00-5,00$ & a & \\
\hline Spannweite & 6 & 9 & 2 & 2 & \\
\hline Min.-Max. & $1-7$ & $1-10$ & $3-5$ & $4-6$ & \\
\hline Soziale Kontakte (n) & 19 & 9 & 1 & 2 & 31 \\
\hline Median & 1,00 & 1,50 & 0,00 & 1,00 & \\
\hline IQR & $0,25-2,00$ & $0,75-2,25$ & $0,00-0,75$ & a & \\
\hline Spannweite & 6 & 3 & 1 & 2 & \\
\hline Min.-Max. & $0-6$ & $0-3$ & $0-1$ & $0-2$ & \\
\hline Werte $(n)$ & 41 & 16 & 10 & 5 & 72 \\
\hline Median & 2,00 & 2,50 & 2,50 & 2,50 & \\
\hline IQR & $1,00-6,75$ & $1,75-4,00$ & $1,25-3,75$ & a & \\
\hline Spannweite & 10 & 3 & 3 & 1 & \\
\hline Min.-Max. & $0-10$ & $1-4$ & $1-4$ & $2-3$ & \\
\hline Lebensort (n) & 7 & 14 & 4 & 5 & 30 \\
\hline Median & 0,00 & 2,00 & 0,50 & 2,50 & \\
\hline IQR & $0,00-1,00$ & $1,00-3,00$ & $0,00-2,50$ & a & \\
\hline Spannweite & 2 & 5 & 3 & 3 & \\
\hline Min.-Max. & $0-2$ & $1-6$ & $0-3$ & $1-4$ & \\
\hline Gesamt & 189 & 96 & 48 & 44 & 377 \\
\hline
\end{tabular}

$n$ Anzahl der dokumentierten Präferenzen, IQR Interquartilsabstand, Min.-Max. Minimum und Maximum

${ }^{a}$ Aufgrund der Anzahl der Dokumentationen ist keine Ausweisung des IQR möglich

\section{Diskussion und Ausblick}

Die durchgeführte explorative Studie untersuchte die Dokumentation von Präferenzen in verschiedenen pflegerischen Settings. Dabei standen die Identifikation, um welche Präferenzen es sich dabei handelt, sowie deren Anzahl und die anhand der quantitativen Daten durchgeführte Kontrastierung der verschiedenen Settings im Fokus.

In den ausgewählten 24 Pflegedokumentationen aus den 4 verschiedenen pflegerischen Settings wurden von den Interviewpartnern/Interviewpartnerinnen insgesamt 377 dokumentierte Aussagen zu Präferenzen identifiziert. Insgesamt konnten die Aussagen 20 verschiedenen Themen zugeordnet werden, aus denen sich 5 Kategorien ergaben: Pflege und Alttag, Freizeit und Geselligkeit, soziale Kontakte, Werte und Lebensort.

Die am häufigsten dokumentierten Präferenzen $(n=149)$ konnten der Kategorie Pflege und Alltag zugeordnet werden. Deutlich seltener konnten Präferenzen zu den Kategorien soziale Kontakte $(n=31)$ und Lebensort $(n=30)$ in der Dokumentation identifiziert werden. Dass der quantitativ deutlich höhere Anteil an dokumentierten Aussagen der Kategorie Pflege und Alltag zugeordnet wurde, könnte auf verschiedene Faktoren zurückzuführen sein. Eine mögliche Erklärung könnte darin bestehen, dass die Dokumentation von Präferenzen aufgrund von externen Anforderungen an die Pflegedokumentation zurückzuführen ist. So könnte es sein, dass eine dokumentierte Präferenz zur Ernährung (wie z.B. „mag herzhaftes Essen auch zum Frühstück und Hausmannskost" oder auch ,... trinkt gerne viel Kaffee“) in unmittelbarem Bezug zur Qualitätsprüfrichtlinie (MDS 2007) und zu der Frage „Werden die individuellen Wünsche zum Essen und Trinken im Rahmen der vereinbarten Leistungserbringung berücksichtigt?" steht. Somit würde dieser Präferenz (in erster Linie) eine hohe Aufmerksamkeit geschenkt, um der Erfüllung einer extern definierten Qualitätsanforderung zu entsprechen und nicht, weil dies den Menschen mit Pflegebedarf wichtig ist. Dies lässt sich auch für weitere Präferenzen aus der Kategorie Pflege und Alltag sowie Freizeitgestaltung feststellen, da auch hier- 
zu explizite Nachfragen im Rahmen der jährlichen Qualitätsprüfungen (MDS 2007) erfolgen. Überraschend war, dass in den Pflegedokumentationen der stationären Langzeitpflege, im Vergleich zu den anderen Settings, nur wenige Nennungen in der Kategorie Pflege und Alltag identifiziert wurden. Möglicherweise ergibt sich dies aus externen sowie institutionellen Rahmenbedingungen, die individualisierten pflegerischen Handlungsabläufen konträr gegenüberstehen (Persson und Wästerfors 2009; Cohen-Mansfield et al. 1995). Dies könnte auch Auswirkungen auf die Dokumentationspraktiken der professionell Pflegenden haben und somit zu einer Verzerrung der Ergebnisse beitragen.

Für die durchgeführte Studie bestanden diverse Limitationen, die womöglich Einfluss auf die Ergebnisse hatten. So ist die geringe Stichprobengröße zu nennen, die sich insbesondere in einzelnen Settings, wie z.B. der Wohngruppe oder der Tagespflege verdeutlicht. Diesbezüglich ist auf den explorativen Charakter der Studie zu verweisen, und das Ziel, einen ersten Einblick in die Dokumentationspraktiken, bezogen auf die Präferenzen des alltäglichen Lebens, zu erhalten. Darüber hinaus kann nicht ausgeschlossen werden, dass besonders detaillierte bzw. wenig detaillierte Dokumentationen in der zufälligen Stichprobenziehung Berücksichtigung fanden. Dies könnte zu Verzerrungen im Bereich der qualitativen als auch der quantitativen Auswertung und somit bei der vorgenommenen Kontrastierung geführt haben. Daneben ist zu erwähnen, dass für die Datenerhebungen die Interviews nicht mithilfe von $\mathrm{Au}-$ diogeräten aufgezeichnet wurden. Dies kann dazu geführt haben, dass Informationen in der Gesprächssituation von den Forschenden nicht berücksichtigt wurden. Allerdings waren bei den Interviews immer 2 Forschende anwesend, sodass einer das Gespräch führen konnte und der Zweite die vorgelesenen Inhalte mithilfe des Tablets schriftlich erfasst hat. Abschließend ist noch zu erwähnen, dass in den Erhebungen eine Vielzahl an weiteren Präferenzen von den professionell Pflegenden mündlich mitgeteilt wurde, welche nicht in der Dokumentation verschriftlicht waren. Diese wurden nicht erfasst und folglich auch nicht in der Auswertung berücksichtigt. Dies zeigt jedoch auf, dass die mündliche Überlieferung von pflegerelevanten Informationen für die professionell Pflegenden nach wie vor eine wesentliche Methode darstellt. Dies ist im Rahmen der Analyse von Pflegedokumentationen zu berücksichtigen.

Die Ergebnisse dieser Studie können erste Hinweise auf Schwierigkeiten hinsichtlich der Integration der Erfassung von Präferenzen in den Pflegeprozess liefern. Dies könnte für die von der Stiftung Wohlfahrtspflege finanzierte Pilotstudie „Präferenzen des täglichen Lebens von alten Menschen - Übersetzung und Pilotierung “ (2017-2020) (Stacke et al. 2020a, b) und für eine ausstehende Implementierung hilfreich sein. Darüber hinaus scheint die Dokumentation von Präferenzen des alltäglichen Lebens von den profes- sionell Pflegenden und ihrer Sensibilität für dieses Thema sowie von dem Vorgehen (unsystematisch und unstrukturiert) bei der Erfassung abhängig zu sein. Bezugnehmend hierzu könnte die Etablierung eines Instruments zur strukturierten Erfassung von Präferenzen zu einer stärkeren Sensibilisierung der Pflegenden für das Thema der Präferenzen von Menschen mit Pflegebedarf und ihrer Berücksichtigung bei der Versorgung führen.

Funding Open Access funding enabled and organized by Projekt DEAL.

\section{Einhaltung ethischer Richtlinien}

Ethische Standards Bei der durchgeführten Studie handelt es sich um die Erfassung und Auswertung von Sekundärdaten. Gemäß der Leitlinie Gute Praxis Sekundärdatenanalyse (AGENS 2014) war die Konsultation einer Ethikkommission für das Forschungsvorhaben nicht erforderlich. Von den professionellen Pflegenden wurde eine informierte Einwilligung für die Studie eingeholt.

Open Access Dieser Artikel wird unter der Creative Commons Namensnennung 4.0 International Lizenz veröffentlicht, welche die Nutzung, Vervielfältigung, Bearbeitung, Verbreitung und Wiedergabe in jeglichem Medium und Format erlaubt, sofern Sie den/die ursprünglichen Autor(en) und die Quelle ordnungsgemäß nennen, einen Link zur Creative Commons Lizenz beifügen und angeben, ob Änderungen vorgenommen wurden.

Die in diesem Artikel enthaltenen Bilder und sonstiges Drittmaterial unterliegen ebenfalls der genannten Creative Commons Lizenz, sofern sich aus der Abbildungslegende nichts anderes ergibt. Sofern das betreffende Material nicht unter der genannten Creative Commons Lizenz steht und die betreffende Handlung nicht nach gesetzlichen Vorschriften erlaubt ist, ist für die oben aufgeführten Weiterverwendungen des Materials die Einwilligung des jeweiligen Rechteinhabers einzuholen.

Weitere Details zur Lizenz entnehmen Sie bitte der Lizenzinformation auf http://creativecommons.org/licenses/by/4.0/deed.de.

\section{Literatur}

Abbott KM, Heid AR, Kleban M, Rovine MJ, Van Haitsma K (2018) The change in nursing home residents' preferences over time. J Am Med Dir Assoc 19(12):1092-1098. https://doi.org/10.1016/ j.jamda.2018.08.004

AGENS (2014) Gute Praxis Sekundärdatenanalyse (GPS). Leitlinie und Empfehlungen

Beikirch E, Breloer-Simon G, Rink F, Roes M (2014) Abschlussbericht Projekt „Praktische Anwendung des Strukturmodells - Effizienssteigerung der Pflegedokumentation in der ambulanten und stationären Langzeitpflege“. BMG, Berlin, Witten

Brummel-Smith K, Butler D, Frieder M, Gibbs N, Henry M, Koons E, Loggers E, Porock D, Reuben DB, Saliba D, Scanlos WJ, Tabbush V, Tinetti M, Tumlinson A, Vladeck BC (2016) Person-centered care: a definition and essential elements. J Am Geriatr Soc 64(1):15-18

Cohen-Mansfield J, Jensen B (2007) Changes in habits related to selfcare in dementia: the nursing home versus adult day care. Am J Alzheimers Dis Other Demen 22:184-189

Cohen-Mansfield J, Werner P, Weinfield M, Braun J, Kraft G, Gerber B, Willens S (1995) Autonomy for nursing home residents: the role of regulations. Behav Sci Law 13:415-423 
Eberhardt D (2018) Personenzentrierung als gelebte Praxis. PADUA 13(1):5

Gordon J, Watts G (2011) Applying skills and knowledge: principle of nursing practice F. Nurs Stand 25:35-37

Van Haitsma K, Curyto K, Spector A, Towsley G, Kleban M, Carpenter B, Ruckdeschel K, Feldman PH, Koren MJ (2013) The preferences for everyday living inventory: scale development and description of psychosocial preferences responses in communitydwelling elders. Gerontologist 53(4):582-595. https://doi.org/10. 1093/geront/gns 102

Van Haitsma KS, Curyto K, Abbott KM, Towsley GL, Spector A, Kleban M (2015) A randomized controlled trial for an individualized positive psychosocial intervention for the affective and behavioral symptoms of dementia in nursing home residents. J Gerontol B Psychol Sci Soc Sci 70(1):35-45. https://doi.org/10.1093/geronb/ gbt 102

Housen P, Shannon GR, Simon B, Edelen MO, Cadogan MP, Jones M, Buchanan J, Saliba D (2009) Why not just ask the resident? J Gerontol Nurs 35(11):40-49. https://doi.org/10.3928/0098913420091001-01

Kesselring A (2013) Die Menschen kennen, die wir pflegen. Krankenpflege 106:14-17

Koren MJ (2010) Person-centered care for nursing home residents: the culture-change movement. Health Aff (Millwood) 29(2):312-317. https://doi.org/10.1377/hlthaff.2009.0966

Krohwinkel M (2013) Fördernde Prozesspflege mit integrierten ABEDLs. Forschung, Theorie und Praxis, Bd. 1. Huber, Bern

MDS (2007) Grundlagen der Qualitätsprüfung nach dem $\S \S 114 \mathrm{ff}$ SGB XI. MDS, Essen

Morgan DL (1993) Qualitative content analysis: a guide to paths not taken. Qual Health Res 3(1):112-121. https://doi.org/10.1177/ 104973239300300107
Persson T, Wästerfors D (2009) "Such trivial matters:" how staff account for restrictions of residents' influence in nursing homes. J Aging Stud 23(1):1-11. https://doi.org/10.1016/j.jaging.2007. 09.005

Saliba D, Buchanan J (2008) Development and validation of a revised nursing home assessment tool; MDS 3.0. Rand Corporation Health, Santa Monica

Saliba D, Jones M, Streim J, Ouslander J, Berlowitz D, Buchanan J (2012) Overview of significant changes in the minimum data set for nursing homes version 3.0. J Am Med Dir Assoc 13:595-601

Simmons SF, Schnelle JF (2004) Individualized feeding assistance care for nursing home residents: Staffing requirements to implement two interventions. J Gerontol 59:M966-M973

Stacke TI, Bergmann JM, Strobel AM, Muller-Widmer R, Purwins D, Manietta C, Rommerskirch M, Nebowsky AE, Wegner A, White J, Kelleter H, Ralic N, Van Haitsma K, Roes M (2020a) Preferences for everyday living inventory (PELI): study protocol for piloting a culture-sensitive and setting-specific translated instrument in German care settings (PELI-D). BMJ Open 10(1):e30268. https://doi.org/10.1136/bmjopen-2019-030268

Stacke TI, Manietta C, Bergmann JM, Purwins D, Rommerskirch M, Roes M (2020b) Übersetzung und kultursensitive Anpassung des PELI (Preferences for Everyday Living Inventory) für den Einsatz in verschiedenen pflegerischen Settings. Pflege (Under Review)

Thompson D, Smith D (1998) Continence restoration in the cognitively impaired adult. Geriatr Nurs 19:87-90

Hinweis des Verlags Der Verlag bleibt in Hinblick auf geografische Zuordnungen und Gebietsbezeichnungen in veröffentlichten Karten und Institutsadressen neutral. 